\title{
A paradigm shift for the prevention and treatment of individual and global obesity
}

\section{Arnold H Slyper \\ Clalit Health Organization, Jerusalem 95 I 4622, Israel}

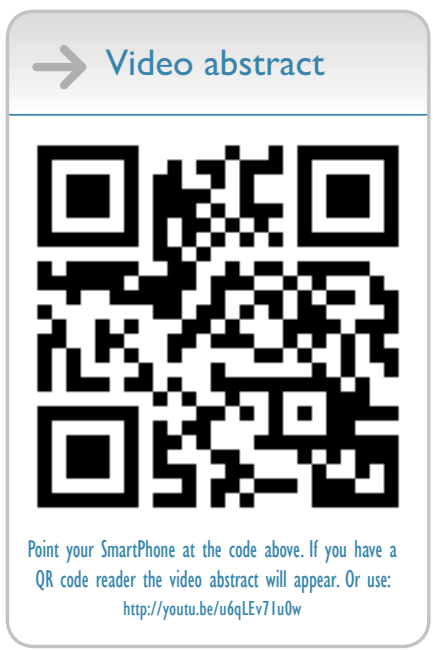

Correspondence: Arnold H Slyper Clalit Health Organization, 22 Bnei Brit St, Jerusalem 95I4622, Israel

Tel +97258578 8844

Email arnoldslyper@yahoo.com
This article was published in the following Dove Press journal: Diabetes, Metabolic Syndrome and Obesity:Targets and Therapy

\begin{abstract}
The prevalence of obesity and overweight has plateaued in developed countries, although at high levels, but in most parts of the world, it continues to increase. Current recommendations for preventing and treating obesity are based mainly on the notion that overeating results from hedonic eating as a result of unlimited access to palatable foods, particularly those high in sugar and fat, and that hedonic centers are able to "override" the body's homeostatic mechanisms. This article proposes that the homeostatic mechanisms affecting appetite and satiety are more important in chronic overeating, and that sufficient evidence exists for adopting a new paradigm for controlling individual and global obesity based on controlling energy homeostasis via the enteroendocrine and gut microbiota systems. Many obese children and adolescents have chronic hunger, supporting the notion that they have a homeostatic rather than hedonic abnormality. The effectiveness of weight loss drugs and bariatric surgery suggests that the brain centers controlling energy homeostasis are able to override centers controlling hedonic drives. Energy homeostasis can also be influenced by nutrition, in particular, by avoiding sweetened drinks and consuming whole grains, vegetables, fruits and other foods that are high in dietary fiber, and thereby influence appetite and satiety. New recommendations are outlined for preventing and treating individual and global obesity based on a paradigm that targets appetite and satiety.
\end{abstract}

Keywords: obesity, appetite, satiety, nutrition

\section{A paradigm shift for the prevention and treatment of obesity}

The prevalence of obesity and overweight has plateaued in developed countries, albeit at high levels, but continues to rise in many parts of the world. ${ }^{1,2}$ Current recommendations for preventing and treating obesity are mainly based on the notion that overeating results from hedonic eating as a result of unlimited access to palatable foods, particularly those high in sugar and fat, and that hedonic centers are able to "override" the body's homeostatic mechanisms. ${ }^{3}$

It is undeniable that hedonic mechanisms can lead to episodes of overeating. This article proposes, however, that the homeostatic mechanisms affecting appetite and satiety are more important in chronic overeating and the development of obesity. Although the physiology underlying these homeostatic mechanisms is still not fully understood, sufficient evidence exists for adopting a new paradigm for individual and global weight control that is directed at the enteroendocrine and gut microbiota systems controlling energy homeostasis. ${ }^{4-6}$ 


\section{Obesity as a chronic disorder of hunger and satiety}

Two types of brain centers regulate food intake. Short- and long-term energy homeostasis is controlled by centers in the hypothalamus and brainstem that receive hormonal and vagal signals relating to the body's metabolic status and the nutrient content of individual meals. The hormones cholecystokinin, glucagon-like peptide (GLP-1), peptide tyrosine tyrosine (PYY) and nesfatin-1, derived from the gastrointestinal tract, have local and central actions and inhibit food intake by crossing the blood-brain barrier and targeting the arcuate nucleus of the hypothalamus. The gastrointestinal-derived peptide ghrelin has peripheral and central actions that stimulate food intake. Messages from the vagus nerve reach the solitary nucleus in the medulla oblongata via the solitary tract, and nerve fibers then project from this nucleus to other areas of the brain. Non-homeostatic hedonic aspects of eating are controlled by centers in the cortex, basal ganglia and limbic system that involve reward-motivated, cognitive and emotional factors in response to visual, olfactory and orosensory cues. ${ }^{\text {? }}$ Both types of centers communicate with each other via neural pathways and both respond to endocrine signals. ${ }^{3,4,8,9} \mathrm{~A}$ magnetic resonance imaging study on sucrose ingestion has suggested greater connectivity between homeostatic and hedonic centers in the obese than in the lean, indicating the possibility that hedonic mechanisms may be enhanced in the obese in the presence of an underlying homeostatic abnormality. ${ }^{10}$

Obese individuals eat larger meals than people of normal weight. ${ }^{11-13}$ Evidence that this is often due to a persistent hyperphagic state comes from a study in the US that provided questionnaires to the parents of obese children and adolescents. ${ }^{14}$ The parents were requested that these be completed together with their offspring, and they included questions about the child's hunger on a 5-point scale ("eats less than others" to "eating a lot, always abnormally hungry") and speed of eating on a 4-point scale ("very slow" to "very fast").

Of the 127 obese patients with a body mass index (BMI) greater than the 95 th percentile, $62.2 \%$ reported "eating a lot and always abnormally hungry" or "eating a lot and often abnormally hungry" compared to $21.4 \%$ of normal weight children with a BMI $<75$ th percentile. Also, $55.7 \%$ of the obese patients admitted to eating "very fast" or "moderately fast" vs $23.3 \%$ of controls. Both differences were highly significant $(P<0.001)$. Hunger and speed of eating were also highly correlated $(P<0.001)$, suggesting that eating fast was part of this hyperphagic state.

This study was repeated in a weight control clinic for children and adolescents in Jerusalem, Israel, and the results were very similar. Of 100 Jewish and Arab obese pediatric patients, $66 \%$ had hunger recognized by the families as abnormal on a 7-point scale and $45 \%$ of these 100 patients had the highest rating, that is, "hunger for most of the time" (AH Slyper, personal communication, November 2018). Hunger of this degree is quite unusual in normal weight children, being seen in only 3 of 71 normal weight controls, and is far more indicative of a persistent homeostatic abnormality than an intermittent hedonic one.

It could be argued that hunger is too subjective a phenomenon to be evaluated meaningfully by a questionnaire. However, the parents' answer to this question reflected their perceptions of how their child usually behaves in relation to food and can be regarded as reasonably objective. The studies in the two countries were performed in community pediatric endocrine centers and the children seen were representative of obese children in these communities.

\section{Changing energy homeostasis by drugs and surgery}

It is evident that weight loss can be achieved by changing the hormonal milieu of the gut, and the evidence for this comes from the use of anorexic drugs and bariatric surgery.

Energy intake is influenced by a number of hormones, including ghrelin which increases the food intake and is secreted predominantly by cells in the stomach, anorexigenic hormones secreted throughout the gut that decrease appetite by means of endocrine and locally acting signals as well as stimulation of the vagus nerve, leptin secreted by the adipose stores and insulin manufactured in the pancreas. Two hormones important in appetite suppression that have been extensively studied are GLP-1 and PYY. Both are secreted by enteroendocrine L-cells found in increasing density from the proximal small intestine to the distal part of the colon. Blood levels of these hormones increase after food ingestion..$^{15,16}$ Both hormones suppress appetite and reduce meal size when injected intravenously. ${ }^{15}$ GLP-1 is also an incretin and may be responsible for almost half of postprandially secreted insulin. ${ }^{17}$

Metformin is commonly used as a first-line treatment for diabetes, and it has mild weight control properties through its ability to influence appetite. It is commonly used by pediatric endocrinologists off-label to control excessive weight gain. ${ }^{18,19}$ Blood glucose lowering occurs only when the drug is administered orally and not intravenously, suggesting that this effect is mediated within the gastrointestinal tract. The reason for the anorexic effect of metformin has not been extensively studied, although the drug does lead to increased fasting levels of GLP-1 in adult non-diabetics and diabetics, independent of changes in glycemia and adiposity. ${ }^{20}$ 
Other classes of drugs that produce weight loss and are approved for adults are GLP-1 analogs and the dipeptidylpeptidase-IV inhibitors that prevent the catalytic breakdown of GLP-1. ${ }^{21}$ The 56-week Scale Obesity and Prediabetes Study found that the GLP-1 analog liraglutide produces a body weight reduction in obese adults of $8.0 \%$ compared to $2.6 \%$ for placebo. ${ }^{22}$ In the SCALE Diabetes randomized controlled trial, $54 \%$ of patients achieved a weight loss of $5 \%$ or more with liraglutide compared to $21 \%$ in the placebo group. ${ }^{23}$

Much of the weight loss achieved after bariatric surgery may well be due to an enteroendocrine effect. Diabetics experience a rapid improvement in blood sugars after surgery and well before significant weight loss occurs, suggesting the incretin effect of GLP-1. ${ }^{24}$ Within a week of Roux-en-Y gastric bypass surgery, meal-induced insulin and GLP-1 secretion increases, the latter as much as tenfold..$^{25} \mathrm{~A}$ sharp rise in postprandial hormones, including GLP-1 and peptide YY, also occurs following vertical sleeve gastrectomy. ${ }^{26}$ Significant changes in food choices, taste functions, hedonic evaluation and self-control also occur following bariatric surgery, and one can speculate that this may also be a consequence of hormonal changes. ${ }^{27}$

Whether changes in the enteroendocrine environment are etiologically related to weight loss with metformin and bariatric surgery are still uncertain. Nevertheless, a number of conclusions can be made from the effectiveness of weight loss drugs and bariatric surgery: 1) the brain centers controlling energy homeostasis can "override" those controlling "hedonic" drives; 2) the enteroendocrine system is an excellent target for influencing energy homeostasis, appetite and satiety and 3) it is possible that brain hedonic centers are in "overdrive" in the obese as a result of disordered energy homeostasis. ${ }^{10}$ This means that for many obese individuals, overeating results from a disorder in physiology and not gluttony.

\section{Changing energy homeostasis via nutrition}

The foods we eat are also able to influence energy homeostasis at the level of the gut. Enteroendocrine secretion is stimulated by the release of the breakdown products of fat, protein and carbohydrate. Fatty acids with $>12$ carbons and partially hydrolyzed proteins stimulate the release of the anorexigenic hormone cholecystokinin from the duodenum and proximal jejunum. ${ }^{4,16}$ Dietary fat has only a weak effect on satiation. ${ }^{28}$ High-protein snacks such as yogurt have been shown to improve appetite and satiety and reduce subsequent food intake. ${ }^{29}$ However, increasing animal protein and fat at the expense of carbohydrate in low- or moderate-carbohydrate diets increases mortality and will not be discussed further in this article. ${ }^{30}$ Most of the satiety effect of carbohydrate comes from its content of dietary fiber, and gut bacteria have a role in this satiety effect. There are about as many bacteria in the gut as cells in the body. ${ }^{31,32}$ About 1,000 different species of bacteria have been identified in the gut, and there is large interindividual variability.

\section{The appetite-suppressing effect of dietary fiber}

The definition of dietary fiber has been much debated, but a broad definition is carbohydrate polymers that escape digestion and absorption in the upper human intestine. The behavior of fiber during digestion is dependent on its physicochemical properties, and this includes particle size, solubility, hydration properties and viscosity.$^{33}$ Based on its solubility in water, dietary fiber is frequently classified as soluble fiber (which includes pectins, gums, mucilages and storage polysaccharides) and insoluble fiber (which includes cellulose, hemicelluloses and lignin).

Fiber delays hunger and increases satiety by slowing gastric emptying. ${ }^{34}$ Fiber also undergoes partial fermentation by gut bacteria into mainly acetate, propionate and butyrate, resulting in high concentrations in the colon. These shortchain fatty acids are a source of energy (about $10 \%$ of the energy from the diet), and via the enteroendocrine cell receptors free fatty acid receptor 1 and free fatty acid receptor 2, they promote the release of GLP-1 and peptide YY, thereby slowing intestinal transit time and suppressing appetite. ${ }^{35}$ In experiments in mice, administration of butyrate and propionate induced gut hormone secretion and reduced food intake. ${ }^{36}$ Targeted administration of propionate to the colon in obese volunteers led to enhanced PYY and GLP-1 secretion and significantly reduced adiposity and weight gain. ${ }^{37}$

As might be anticipated, dietary fiber has been shown to significantly influence hunger and satiety. In the following studies, hunger and satiety were measured by psychological instruments such as analog scales, and these have been shown to be reliable for appetite research. ${ }^{38}$ Howarth et a ${ }^{39}$ reported on 38 studies that examined the influence of fiber on hunger or satiety in healthy subjects under conditions of fixed energy intake, and the majority showed that fiber increased satiety between meals. In a review of 12 interventional studies, it was concluded that a $14 \mathrm{~g}$ per day increase in fiber in an ad libitum diet resulted in a $10 \%$ decrease in energy intake and $1.9 \mathrm{~kg}$ decrease in body weight over 3.8 months. Energy intake was 
suppressed more and weight loss was greater in overweight patients. From a review of 66 long-term studies, Wanders et $\mathrm{al}^{40}$ concluded that fiber intake was associated with an average reduction in body weight of $0.4 \%$ over 4 weeks, and that weight loss was unrelated to any physicochemical properties of the fiber. A review by Pereira and Ludwig ${ }^{41}$ noted that fiber had a positive influence on hunger and satiety in 17 studies, showed mixed results in 7 and had no effect in 3 . Wanders et $\mathrm{al}^{40}$ discuss that soluble fiber characterized as more viscous and with greater resistance to flow, such as from pectins, $\beta$-glucans and guar gum, reduces appetite more effectively than less viscous fibers, with the more viscous fibers resulting in a $59 \%$ reduction in appetite compared to a $14 \%$ reduction from the less viscous fibers.

Microbial richness and biodiversity in the gut is greater in the lean than in the obese, and a lean pattern is associated with greater short-chain fatty acid production. ${ }^{32,42}$ Microbial richness and biodiversity is seen in populations with a high dietary fiber intake compared to those with a low intake, and in this study was associated with higher fecal short-chain fatty acid concentrations. ${ }^{43}$ Switching between a vegetarian and animal-based diet rapidly changes the composition of the stool microbiome. ${ }^{44}$ This raises the possibility of a dynamic interplay between nutrition and the gut flora that could amplify the benefits of a fiber-rich diet.

\section{The obesogenic effect of naturally sweetened beverages}

Naturally sweetened drinks are an important cause of weight gain. In a meta-analysis, Te Morenga et $\mathrm{al}^{45}$ analyzed 30 randomized trials and showed that for adults on ad libitum diets (ie, without strict control of food intake), an increased intake of dietary sugars was associated with an increase in body weight of $0.75 \mathrm{~kg}(0.30-1.19, P=0.001)$ over several weeks. On the other hand, in trials of diets high in free sugars compared to diets low in free sugars in which there was isocaloric substitution of sugars for other carbohydrates, there was no significant change in body weight. In 38 cohort studies, the OR of becoming overweight or obese increased to $1.55(1.32-1.82)$ among groups with the highest intake of sugar-sweetened beverages compared to those with the lowest intake, after 1 year of follow-up. ${ }^{41}$ In a review and meta-analysis, Malik et $\mathrm{al}^{46}$ found that one daily serving increment of a sugar-sweetened beverage in cohort studies was associated with a 0.06 (95\% CI: $0.02,0.10)$ and 0.05 (95\% CI: 0.03, 0.07)-unit increase in BMI in children and $0.22 \mathrm{~kg}(95 \% \mathrm{CI}: 0.09,0.34 \mathrm{~kg})$ and $0.12 \mathrm{~kg}$ (95\% CI: 0.10 , $0.14 \mathrm{~kg}$ ) weight gain in adults over 1 year in random- and fixed-effects models, respectively. Randomized controlled trials in adults demonstrated increases in body weight when sugar-sweetened beverages were added $(0.85 \mathrm{~kg}$; $95 \% \mathrm{CI}$ : $0.50,1.20 \mathrm{~kg}$ ), and trials in children showed reduction in BMI gain when sugar-sweetened beverages were reduced. In a meta-analysis of cohort studies, $\mathrm{Hu}$ and $\mathrm{Fb}^{47}$ found that a higher intake of sugar-sweetened beverages among children was associated with a 55\% (95\% CI 32\%-82\%) greater risk of becoming overweight or obese compared to those with lower intake. By contrast, the association between yearly weight gain and intake of solid energy-dense snack foods such as sweets, cakes, pastries and savory snacks is small, inconsistent and no different from that of other refined carbohydrates. ${ }^{48,49}$

The simplest explanation for the weight-promoting effect of sugar-sweetened drinks is that they have little satiety value and this leads to failure to adjust for the energy supplied by these beverages at subsequent eating. ${ }^{50}$ In support of this is that glucose and fructose when administered as intra-gastric infusions lead to the secretion of GLP-1, peptide YY and ghrelin, but their satiety and fullness effects are little different from that of water. ${ }^{51}$ On the other hand, it cannot be excluded that the situation is more complex than this. In obese and lean women, there is greater activity in brainstem vagal pathways after consuming high-sucrose-containing beverages compared to low-sucrose-containing beverages, possibly indicating that energy homeostatic mechanisms are also involved in the response to sugar-containing drinks. ${ }^{10}$

\section{Conclusion}

On the basis of this discussion, it is possible to speculate why the US population has experienced such marked weight gain over the last few decades. The campaign that was initiated in the 1960s to decrease consumption of saturated fat led to an increased intake of carbohydrate as a percentage of total calories. Because of the focus on quality and quantity of fat, attention was diverted away from the quality of carbohydrate. These two factors resulted in a greater consumption of processed carbohydrates and sugars. Full fat milk, a satietyinducing drink due to its protein and fat content, came to be regarded as a suspect food because of its content of saturated fat and was replaced by low-fat milks and sugar-containing soft drinks. ${ }^{52}$ Eggs, also a filling food, were cut back because of their cholesterol content. ${ }^{53}$ These changes resulted in low-fiber, low-satiety diets that encouraged overeating as a result of their influence on homeostatic mechanisms and not because of their hedonic value. The adoption of Western-type foods throughout much of the developing world has similarly led to diets that encourage people to overeat. 


\section{Recommendations}

On the basis of this discussion, a new paradigm is suggested for individual and global weight control:

Optimizing satiety needs to be the initial step for weight control or weight loss, since calorie restriction is unlikely to succeed over the long term if there is continuing abnormal hunger. To achieve this, sweetened drinks should be eliminated and foods rich in fiber, such as vegetables, fruits and whole grains, should be substituted for refined carbohydrates. This will not only help in preventing and treating obesity but also other conditions such as hyperlipidemia, metabolic syndrome, coronary artery disease, diabetes and hypertension..$^{54,55}$

There is a place for satiety-inducing drugs in the treatment of obese patients who lack the ability to make changes in their diets because of their high hedonic drives, and new drugs acting at the level of the gut or centrally are now available or in the pipeline. ${ }^{56}$ Understandably, no pharmaceutical company has yet sponsored a study looking at how patients can be weaned off their weight-loss drug without regaining weight. Many patients will be permanently hooked on these drugs, unless they are able to make changes in their diet that will improve their appetite and satiety. ${ }^{19}$ Because of this, drug treatment should be used as an aid to dietary treatment and not as a substitute. Admittedly, this is a theoretical construct, as no studies have been reported to date demonstrating that it is actually possible to successfully wean patients off these drugs without regaining lost weight. This information is much needed as more satiety-inducing drugs become available and their use increases.

However, it is the realm of global public health that the impact of this new paradigm should be most apparent. The message to the population needs to be simple and focused. People need to eliminate sweetened drinks and replace refined carbohydrates with natural fiber-rich foods. A practical way of achieving these goals is to place a tax on sweetened drinks, as has been done in the UK, France, Hungry, Mexico, Norway, the United Arab Emirates and some states in the US. ${ }^{57}$ A meta-analysis of nine articles, six from the USA and one each from Mexico, Brazil and France, showed that higher prices for these drinks were associated with a lower demand. ${ }^{58}$ Four articles found an increased demand for alternative beverages such as fruit juice and milk and reduced demand for diet drinks, and the six articles from the USA showed that a higher price led to a decrease in BMI and reduced prevalence of overweight and obesity. The money collected from this tax could be returned to the population by subsidizing high-fiber stables and non-imported vegetables and fruits.
In conclusion, current recommendations are making little dent in individual or global obesity. This new paradigm fits well into what we know about the physiology of hunger and satiety and is supported by nutritional studies. Moreover, the advice to eat vegetables, fruits and whole grains in place of refined carbohydrate and sugars is obvious enough that it should be readily accepted by all populations.

\section{Disclosure}

The author reports no conflicts of interest in this work.

\section{References}

1. Ng M, Fleming T, Robinson M, et al. Global, regional, and national prevalence of overweight and obesity in children and adults during 1980-2013: a systematic analysis for the Global Burden of Disease Study 2013. Lancet. 2014;384(9945):766-781.

2. NCD Risk Factor Collaboration (NCD-RisC). Worldwide trends in body-mass index, underweight, overweight, and obesity from 1975 to 2016: a pooled analysis of 2416 population-based measurement studies in 128.9 million children, adolescents, and adults. Lancet. 2017;390(10113):2627-2642.

3. Berthoud HR. Metabolic and hedonic drives in the neural control of appetite: who is the boss? Curr Opin Neurobiol. 2011;21(6):888-896.

4. Bodnaruc AM, Prud'homme D, Blanchet R, Giroux I. Nutritional modulation of endogenous glucagon-like peptide-1 secretion: a review. Nutr Metab (Lond). 2016;13:92.

5. Prinz P, Stengel A. Control of food intake by gastrointestinal peptides: mechanisms of action and possible modulation in the treatment of obesity. J Neurogastroenterol Motil. 2017;23(2):180-196.

6. Rebello C, Greenway FL, Dhurandhar NV. Functional foods to promote weight loss and satiety. Curr Opin Clin Nutr Metab Care. 2014;17(6):596-604.

7. Berthoud HR, Lenard NR, Shin AC. Food reward, hyperphagia, and obesity. Am J Physiol Regul Integr Comp Physiol. 2011;300(6):R1266-R1277.

8. Williams DL. Neural integration of satiation and food reward: role of GLP-1 and orexin pathways. Physiol Behav. 2014;136:194-199.

9. Perello M, Dickson SL. Ghrelin signalling on food reward: a salient link between the gut and the mesolimbic system. $J$ Neuroendocrinol. 2015;27(6):424-434.

10. Kilpatrick LA, Coveleskie K, Connolly L, et al. Influence of sucrose ingestion on brainstem and hypothalamic intrinsic oscillations in lean and obese women. Gastroenterology. 2014;146(5):1212-1221.

11. Acosta A, Camilleri M, Shin A, et al. Quantitative gastrointestinal and psychological traits associated with obesity and response to weight-loss therapy. Gastroenterology. 2015;148(3):537-546.e4.

12. Berg C, Lappas G, Wolk A, et al. Eating patterns and portion size associated with obesity in a Swedish population. Appetite. 2009;52(1):21-26.

13. de Castro JM, King GA, Duarte-Gardea M, Gonzalez-Ayala S, Kooshian $\mathrm{CH}$. Overweight and obese humans overeat away from home. Appetite 2012;59(2):204-211.

14. Slyper AH, Kopfer K, Huang WM, Re'em Y. Increased hunger and speed of eating in obese children and adolescents. J Pediatr Endocrinol Metab. 2014;27(5-6):413-417.

15. Gunawardene AR, Corfe BM, Staton CA. Classification and functions of enteroendocrine cells of the lower gastrointestinal tract. Int $J$ Exp Pathol. 2011;92(4):219-231.

16. Steinert RE, Feinle-Bisset C, Asarian L, Horowitz M, Beglinger C, Geary N. Ghrelin, CCK, GLP-1, and PYY(3-36): secretory controls and physiological roles in eating and glycemia in health, obesity, and after RYGB. Physiol Rev. 2017;97(1):411-463. 
17. Nauck M, Stöckmann F, Ebert R, Creutzfeldt W. Reduced incretin effect in type 2 (non-insulin-dependent) diabetes. Diabetologia. 1986;29(1):46-52.

18. Adeyemo MA, McDuffie JR, Kozlosky M, et al. Effects of metformin on energy intake and satiety in obese children. Diabetes Obes Metab. 2015;17(4):363-370.

19. Brufani C, Crinò A, Fintini D, Patera PI, Cappa M, Manco M. Systematic review of metformin use in obese nondiabetic children and adolescents. Horm Res Paediatr. 2013;80(2):78-85.

20. Preiss D, Dawed A, Welsh P, et al; DIRECT consortium group. Sustained influence of metformin therapy on circulating glucagon-like peptide-1 levels in individuals with and without type 2 diabetes. Diabetes Obes Metab. 2017;19(3):356-363.

21. Aroda VR. A review of GLP-1 receptor agonists: evolution and advancement, through the lens of randomised controlled trials. Diabetes Obes Metab. 2018;20 Suppl 1:22-33.

22. Pi-Sunyer X, Astrup A, Fujioka K, et al; SCALE Obesity and Prediabetes NN8022-1839 Study Group. A randomized, controlled trial of 3.0 $\mathrm{mg}$ of liraglutide in weight management. $N$ Engl J Med Overseas Ed. 2015;373(1):11-22.

23. Davies MJ, Bergenstal R, Bode B, et al; NN8022-1922 Study Group. Efficacy of liraglutide for weight loss among patients with type 2 diabetes: the SCALE diabetes randomized clinical trial. JAMA. 2015;314(7):687-699.

24. Hutch CR, Sandoval D. The role of GLP-1 in the metabolic success of bariatric surgery. Endocrinology. 2017;158(12):4139-4151.

25. Salehi M, D'Alessio DA. Mechanisms of surgical control of type 2 diabetes: GLP-1 is the key factor-maybe. Surg Obes Relat Dis. 2016;12(6):1230-1235.

26. Magouliotis DE, Tasiopoulou VS, Sioka E, Chatedaki C, Zacharoulis D. Impact of bariatric surgery on metabolic and gut microbiota profile: a systematic review and meta-analysis. Obes Surg. 2017;27(5):1345-1357.

27. Münzberg H, Laque A, Yu S, Rezai-Zadeh K, Berthoud HR. Appetite and body weight regulation after bariatric surgery. Obes Rev. 2015;16 Suppl 1:77-90.

28. Warrilow A, Mellor D, McKune A, Pumpa K. Dietary fat, fibre, satiation, and satiety - a systematic review of acute studies. Eur J Clin Nutr. Epub 2018 Aug 30.

29. Ortinau LC, Hoertel HA, Douglas SM, Leidy HJ. Effects of high-protein vs high- fat snacks on appetite control, satiety, and eating initiation in healthy women. Nutr J. 2014;13:97.

30. Seidelmann SB, Claggett B, Cheng S, et al. Dietary carbohydrate intake and mortality: a prospective cohort study and meta-analysis. Lancet Public Health. 2018;3(9):e419-e428.

31. Blaut M. Gut microbiota and energy balance: role in obesity. Proc Nutr Soc. 2015;74(3):227-234.

32. Davis HC. Can the gastrointestinal microbiota be modulated by dietary fibre to treat obesity? Iran J Med Sci. 2018;187(2):393-402.

33. Capuano E. The behavior of dietary fiber in the gastrointestinal tract determines its physiological effect. Crit Rev Food Sci Nutr. 2017;57(16):3543-3564.

34. Benini L, Castellani G, Brighenti F, et al. Gastric emptying of a solid meal is accelerated by the removal of dietary fibre naturally present in food. Gut. 1995;36(6):825-830

35. Kaji I, Karaki S, Kuwahara A. Short-chain fatty acid receptor and its contribution to glucagon-like peptide-1 release. Digestion. 2014;89(1):31-36.

36. Lin HV, Frassetto A, Kowalik EJ Jr, et al. Butyrate and propionate protect against diet-induced obesity and regulate gut hormones via free fatty acid receptor 3-independent mechanisms. PLoS One. 2012;7(4):e35240.
37. Chambers ES, Viardot A, Psichas A, et al. Effects of targeted delivery of propionate to the human colon on appetite regulation, body weight maintenance and adiposity in overweight adults. Gut. 2015;64(11):1744-1754.

38. Flint A, Raben A, Blundell JE, Astrup A. Reproducibility, power and validity of visual analogue scales in assessment of appetite sensations in single test meal studies. Int JObes Relat Metab Disord. 2000;24(1):38-48.

39. Howarth NC, Saltzman E, Roberts SB. Dietary fiber and weight regulation. Nutr Rev. 2001;59(5):129-139.

40. Wanders AJ, van den Borne JJ, de Graaf C, et al. Effects of dietary fibre on subjective appetite, energy intake and body weight: a systematic review of randomized controlled trials. Obes Rev. 2011;12(9):724-739.

41. Pereira MA, Ludwig DS. Dietary fiber and body-weight regulation. Observations and mechanisms. Pediatr Clin North Am. 2001;48(4):969-980.

42. Human Microbiome Project Consortium. Structure, function and diversity of the healthy human microbiome. Nature. 2012;486(7402):207-214.

43. De Filippo C, Cavalieri D, Di Paola M, et al. Impact of diet in shaping gut microbiota revealed by a comparative study in children from Europe and rural Africa. Proc Natl Acad Sci U SA. 2010;107(33):14691-14696.

44. David LA, Maurice CF, Carmody RN, et al. Diet rapidly and reproducibly alters the human gut microbiome. Nature. 2014;505(7484):559-563.

45. Te Morenga L, Mallard S, Mann J. Dietary sugars and body weight: systematic review and meta-analyses of randomised controlled trials and cohort studies. BMJ. 2012;346:e7492.

46. Malik VS, Pan A, Willett WC, Hu FB. Sugar-sweetened beverages and weight gain in children and adults: a systematic review and metaanalysis. Am J Clin Nutr. 2013;98(4):1084-1102.

47. Hu FB. Resolved: there is sufficient scientific evidence that decreasing sugar-sweetened beverage consumption will reduce the prevalence of obesity and obesity-related diseases. Obes Rev. 2013;14(8):606-619.

48. Hendriksen MA, Boer JM, du H, Feskens EJ, van der A DL. No consistent association between consumption of energy-dense snack foods and annual weight and waist circumference changes in Dutch adults. Am J Clin Nutr. 2011;94(1):19-25.

49. Mozaffarian D, Hao T, Rimm EB, Willett WC, Hu FB. Changes in diet and lifestyle and long-term weight gain in women and men. $N$ Engl $J$ Med Overseas Ed. 2011;364(25):2392-2404.

50. Stanhope KL. Sugar consumption, metabolic disease and obesity: the state of the controversy. Crit Rev Clin Lab Sci. 2016;53(1):52-67.

51. Steinert RE, Frey F, Töpfer A, Drewe J, Beglinger C. Effects of carbohydrate sugars and artificial sweeteners on appetite and the secretion of gastrointestinal satiety peptides. Br J Nutr. 2011;105(9): $1320-1328$.

52. Slyper AH, Huang WM. Milk, dairy fat and body weight in pediatrics: time for reappraisal. Infant Child Adolesc Nutr. 2009;1(3):148-159.

53. Griffin BA. Eggs: good or bad? Proc Nutr Soc. 2016;75(3): 259-264.

54. Slyper AH. The influence of carbohydrate quality on cardiovascular disease, the metabolic syndrome, type 2 diabetes, and obesity - an overview. J Pediatr Endocrinol Metab. 2013;26(7-8):617-629.

55. Streppel MT, Arends LR, van 't Veer P, Grobbee DE, Geleijnse JM. Dietary fiber and blood pressure: a meta-analysis of randomized placebo-controlled trials. Arch Intern Med. 2005;165(2):150-156.

56. Srivastava G, Apovian C. Future pharmacotherapy for obesity: new anti-obesity drugs on the horizon. Curr Obes Rep. 2018;7(2):147-161.

57. Wikipedia. Available from: https://en.wikipedia.org/wiki/Sugary_drink_ tax. Accessed October 25, 2018.

58. Cabrera Escobar MA, Veerman JL, Tollman SM, Bertram MY, Hofman KJ. Evidence that a tax on sugar sweetened beverages reduces the obesity rate: a meta-analysis. BMC Public Health. 2013;13:1072. 
Diabetes, Metabolic Syndrome and Obesity: Targets and Therapy

Diabetes, Metabolic Syndrome and Obesity: Targets and Therapy is an international, peer-reviewed open-access journal committed to the rapid publication of the latest laboratory and clinical findings in the fields of diabetes, metabolic syndrome and obesity research. Original research, review, case reports, hypothesis formation, expert opinion and commentaries are all considered for publication. The manuscript management system is completely online and includes a very quick and fair peer-review system, which is all easy to use. Visit http://www.dovepress.com/testimonials.php to read real quotes from published authors.

Submit your manuscript here: https://www.dovepress.com/diabetes-metabolic-syndrome-and-obesity-targets-and-therapy-journal 